\title{
Case Report of Successful Treatment of Fibrosing Cholestatic Hepatitis C with Sofosbuvir and Ribavirin after Liver Transplantation
}

\author{
${ }^{1}$ The Recanati/Miller Transplantation Institute, Mount Sinai Health \\ System, New York, New York \\ 2 The Lillian and Henry M. Stratton - Hans Popper Department of \\ Pathology, Mount Sinai Health System, New York, New York
}

Brian Kim, MD ${ }^{1}$ Anshu Trivedi, MD $\quad$ Swan N. Thung, MD ${ }^{2}$ Priya Grewal, MD ${ }^{1}$
Address for correspondence Brian Kim, MD, Mount Sinai Health System, 1425 Madison Avenue, 11th Floor, New York, NY 10029 (e-mail: brian.kim@mountsinai.org).

Semin Liver Dis 2014;34:108-112.

\begin{abstract}
Keywords

- hepatitis C virus

- fibrosing cholestatic hepatitis

- cholestatic variant of hepatitis C

- liver transplant

- sofosbuvir

- ribavirin

Fibrosing cholestatic hepatitis is an unusual complication of hepatitis C virus (HCV) recurrence after liver transplant. Fibrosing cholestatic hepatitis is marked by aggressive progression of cholestasis and fibrosis, leading to accelerated graft loss and/or death. Sofosbuvir (GS-7977) is an oral nucleotide analogue inhibitor of HCV polymerase activity. It is a second-generation, direct-acting, antiviral for the treatment of HCV infection. This case illustrates a patient with recurrent HCV with fibrosing cholestatic hepatitis, who was successfully treated with a combination of sofosbuvir and ribavirin with normalization of liver enzyme activities and resolution of HCV-related symptoms. The favorable side effect profile and the lack of drug-drug interaction with immunosuppressive medications make the combination of sofosbuvir and ribavirin a promising regimen for severe HCV recurrence.
\end{abstract}

Hepatitis C virus (HCV) infection is the most common etiology of liver disease requiring liver transplantation (LT) in the United States. ${ }^{1}$ Among patients who have HCV viremia prior to LT, recurrence of HCV and reinfection of the allograft after LT is universal. ${ }^{2}$ HCV recurrence is accelerated in patients after LT secondary to suppression of host immune response. Cirrhosis of the allograft may occur within 5 years of transplant. ${ }^{3} \mathrm{HCV}$ recurrence is the most frequent cause of graft loss and death among patients transplanted for HCVrelated liver complications. ${ }^{4}$ Fibrosing cholestatic hepatitis (FCH), also known as cholestatic variant of hepatitis $\mathrm{C}$, is a particularly aggressive form of HCV recurrence in the allograft that complicates 5 to $14 \%$ of LT performed for $\mathrm{HCV}^{5,6}$ It is characterized by early HCV recurrence with marked cholestasis and fibrosis. Most cases are fatal secondary to rapidly progressive liver dysfunction with graft loss seen within the first 1 to 2 years after LT. ${ }^{7}$ The combination of pegylated interferon (PegINF) and ribavirin has been used with limited success. $^{8}$

Sofosbuvir is a potent, oral nucleotide analog that inhibits $\mathrm{HCV}$ polymerase activity. In combination with ribavirin, sofosbuvir has shown high rates of sustained virological response (SVR) for various genotypes, even in the absence of PegINF. ${ }^{9,10}$

We report a case of FCH in a patient who underwent LT for HCV cirrhosis, who was successfully treated with a combination of sofosbuvir and ribavirin for 24 weeks.

\section{Case Presentation}

A 49-year-old man with history of cirrhosis secondary to HCV and alcohol underwent LT in September 2011.

He was diagnosed with HCV and alcohol-related cirrhosis 10 years prior to LT. Patient denied risk factors for HCV
Copyright $\odot 2014$ by Thieme Medical Publishers, Inc., 333 Seventh Avenue, New York, NY 10001, USA. Tel: +1(212) 584-4662.
DOI http://dx.doi.org/ 10.1055/s-0034-1371084. ISSN $0272-8087$. 
infection, including prior blood transfusions, tattoos, or intravenous drug use. The HCV genotype was $1 \mathrm{~A}$, and the patient's IL28B polymorphism was C/T. The decision was made to hold treatment of his $\mathrm{HCV}$ at that time as his liver disease was well compensated and he had severe thrombocytopenia.

A few months prior to transplantation, the patient developed lower extremity edema, worsening ascites, and jaundice. Patient denied alcohol use. The impression was worsening liver disease in the setting of HCV and alcoholic cirrhosis. The patient was evaluated for LT and placed on the waiting list. Slowly his Models for End-Stage Liver Disease (MELD) score rose to 35, and he received an allograft from a 46-year-old male brain-dead donor who expired from a cerebral vascular accident. The donor liver was noted to have a normal anatomy with no fat or fibrosis on pretransplant frozen-section biopsy. The recipient patient's HCV viral load prior to transplantation was 12,000 RNA IU/mL.

The liver transplantation was unremarkable. Postoperatively, the patient received triple immunosuppressive therapy consisting of tacrolimus, prednisone, and mycophenolic acid, which was changed to tacrolimus monotherapy at 6 months post-LT.

Six months after LT, the patient presented to clinic with mild abdominal discomfort. Blood tests were obtained, and the liver chemistry tests were noted to be markedly abnormal with rise in aspartate aminotransferase (AST) to $310 \mathrm{U} / \mathrm{L}$ (normal 1-50 U/L), alanine aminotransferase (ALT) to $166 \mathrm{U} / \mathrm{L}$ (normal 1-53 U/L), alkaline phosphate (AP) to $252 \mathrm{U} / \mathrm{L}$ (normal $30-110 \mathrm{U} / \mathrm{L}$ ), and gamma-glutamyl transpeptidase (GGT) to $1380 \mathrm{U} / \mathrm{L}$ (normal $10-54 \mathrm{U} / \mathrm{L}$ ). The total and direct bilirubin levels were normal. A magnetic resonance cholangiogram was obtained showing no biliary abnormalities, and the liver did not appear cirrhotic. No steatosis was noted. A transjugular liver biopsy was performed to further evaluate the liver test abnormalities.

The liver allograft showed expansion of most portal tracts by moderate mixed cellular portal inflammation with rare eosinophils. Bile duct damage and endothelialitis were present ( - Fig. 1A). Apoptotic bodies were seen scattered throughout the lobules (-Fig. 1B). Trichrome stain showed no significant fibrosis (-Fig. 1C). The findings were consistent with recurrent hepatitis $C$ and moderate acute cellular rejection. His HCV RNA level was noted to be $3,000,000$ RNA IU $/ \mathrm{mL}$.

Twelve months after LT, the liver chemistry tests remained abnormal. He was now jaundiced and fatigued with some ascites and muscle wasting.

Repeat biopsy showed chronic hepatitis with extensive periportal fibrosis and portal to central bridging fibrous septa with mild to moderate lymphocytic infiltration, moderate interface hepatitis, and mild ductular reaction (-Fig. 2A). Lobules showed centrilobular hepatocellular and canalicular cholestasis and mild necroinflammation (-Fig. 2B). Cytokeratin (CK) 7 immunostain showed bile duct loss in 4 of 16 complete portal tracts (25\%), mild ductular reaction, and rare cholestatic hepatocytes. Trichrome stain confirmed the prominent periportal fibrosis with adjacent pericellular fibrosis and focal portal to central
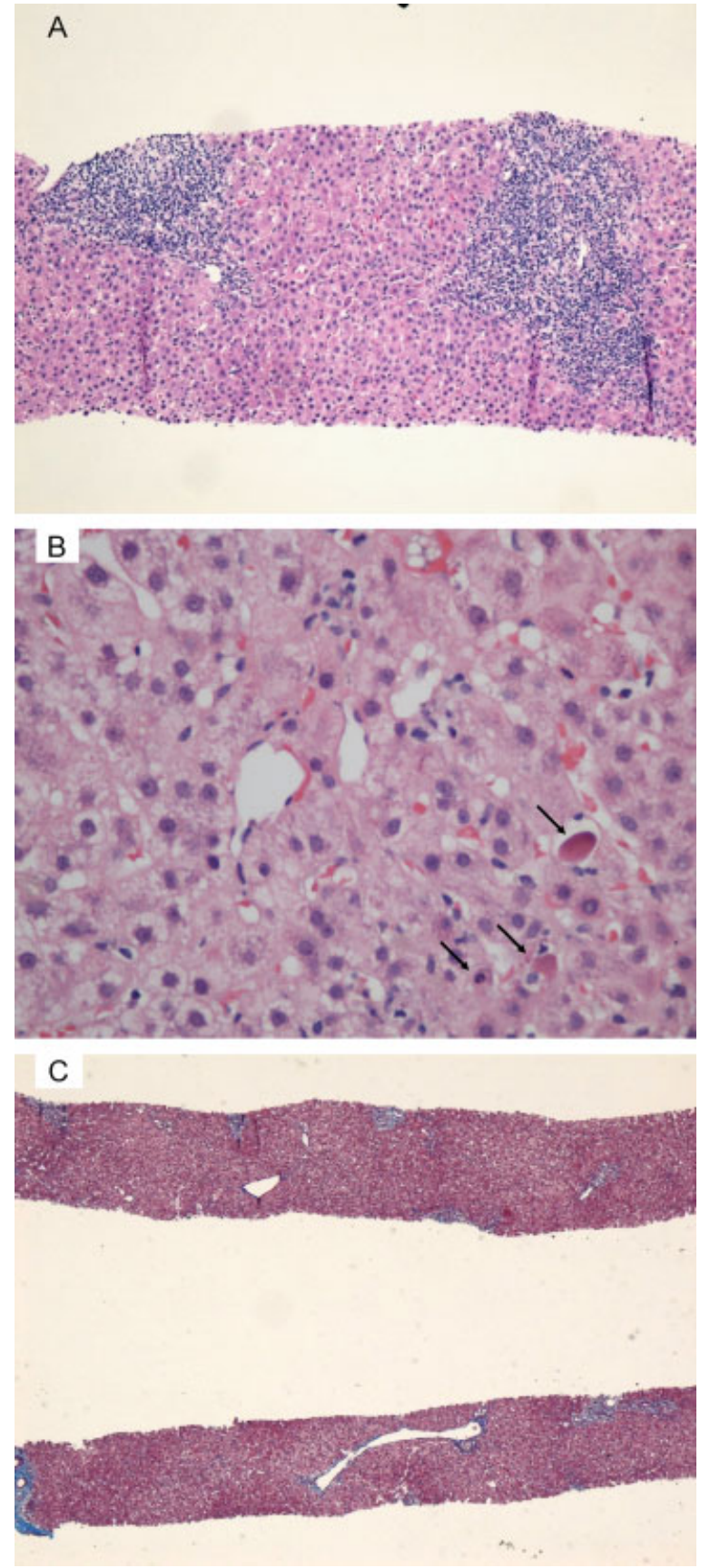

Fig. 1 Liver biopsy 6 months after liver transplantation. (A) Portal tracts with mixed cellular infiltrate, endotheliitis and bile duct damage, consistent with acute rejection (hematoxylin and eosin [H\&E], x100). (B) Mild lobular inflammation and scattered acidophilic bodies (arrows), consistent with recurrent hepatitis C (H\&E, x400). (C) Portal fibrosis and fibrous septa are not present (Trichrome stain, $\mathrm{x} 40$ ).

bridging fibrous septa ( $\mathbf{- F i g} \cdot \mathbf{2 C}$ ). Copper stain was negative. The findings were consistent with cholestatic variant of recurrent hepatitis $\mathrm{C}$ (grade 3 of 4 , stage 2 of 4 ) and possible ongoing chronic rejection. His HCV RNA level was 12,000,000 RNA IU/mL. His tacrolimus trough level was maintained in the range of 7 to $10 \mathrm{ng} / \mathrm{mL}$ to treat any ongoing chronic rejection. 
The combination of reduced-dose PegINF alfa-2a ( $90 \mu \mathrm{g}$ via subcutaneous injection weekly) and reduced-dose ribavirin (200 mg orally twice per day) was started. Treatment was discontinued after 2 weeks as the patient experienced debilitating fatigue and worsening jaundice. His HCV RNA level remained elevated at 8,600,000 RNA IU/mL.

By 15 months after LT, his total bilirubin had risen to $3.8 \mathrm{mg} / \mathrm{dL}$ (normal $0.1-1.2 \mathrm{mg} / \mathrm{dL}$ ), direct bilirubin to $2.6 \mathrm{mg} / \mathrm{dL}$ (normal $0.0-0.8 \mathrm{mg} / \mathrm{dL}$ ), AST to $122 \mathrm{U} / \mathrm{L}$, ALT to $48 \mathrm{U} / \mathrm{L}, \mathrm{AP}$ to $583 \mathrm{U} / \mathrm{L}$, and GGT to $2069 \mathrm{U} / \mathrm{L}$. Repeat biopsy of the liver was performed showing chronic hepatitis with moderate portal inflammation, occasional lymphoid aggregates, moderate interface hepatitis, and moderate lobular

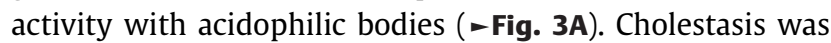
present with feathery degeneration of hepatocytes (-Fig. 3B). Trichrome stain showed extensive periportal and perisinusoidal fibrosis with extensive bridging fibrous septa (-Fig. 3C). Immunostain for CK7 exhibited, moderate ductular reaction, rare cholestatic hepatocytes and 21 of 36 portal tracts contained bile ducts ( - Fig. 3D). The findings were consistent with cholestatic variant of recurrent hepatitis C (grade 3 of 4, stage 3 of 4). The biopsy showed increased necroinflammatory activity and increased fibrosis as compared with the previous biopsy.

An appeal was made to Gilead Sciences (Foster City, CA) for compassionate use of the direct-acting antiviral sofosbuvir (formerly named, GS-7977). The use of the drug was reviewed and approved by The Mount Sinai Medical Center Institutional Review Board (IRB). Sixteen months after LT, the patient began treatment with an interferon-free combination of sofosbuvir (400 mg orally, once per day) and ribavirin (200 mg orally, twice per day). His pretreatment HCV RNA level was 4,000,000 RNA IU/mL.

After 12 weeks of treatment, the HCV RNA was undetectable $(<43 \mathrm{RNA} \mathrm{IU} / \mathrm{mL})$ and remained undetectable until the end of the treatment course at week 24 ( - Fig. 4). His liver chemistry tests began to improve by week 2 and were normal at the end of the treatment course at week 24 (-Fig. 5). During the treatment, the patient did not experience any new side effects. He did not develop anemia or evidence of marrow suppression during the treatment. His platelet count improved during the course of his treatment ( - Fig. 6). During treatment his tacrolimus trough level remained stable and no dose changes were made.

Two weeks after the completion of his HCV treatment, the patient's liver chemistry tests remain normal with persistently undetectable HCV viral load (-Figs. 4, 5). The patient's ascites and fatigue had resolved. He gained $3 \mathrm{~kg}$ during his treatment course.

\section{Discussion}

The treatment of hepatitis $C$ virus infection post-LT is challenging. The combination of pegylated interferon and ribavirin may be attempted, but its use is restricted by severe side effects and limited efficacy. ${ }^{11,12}$ With the addition of first-generation, direct-acting antivirals such as telaprevir or boceprevir, efficacy may increase, but
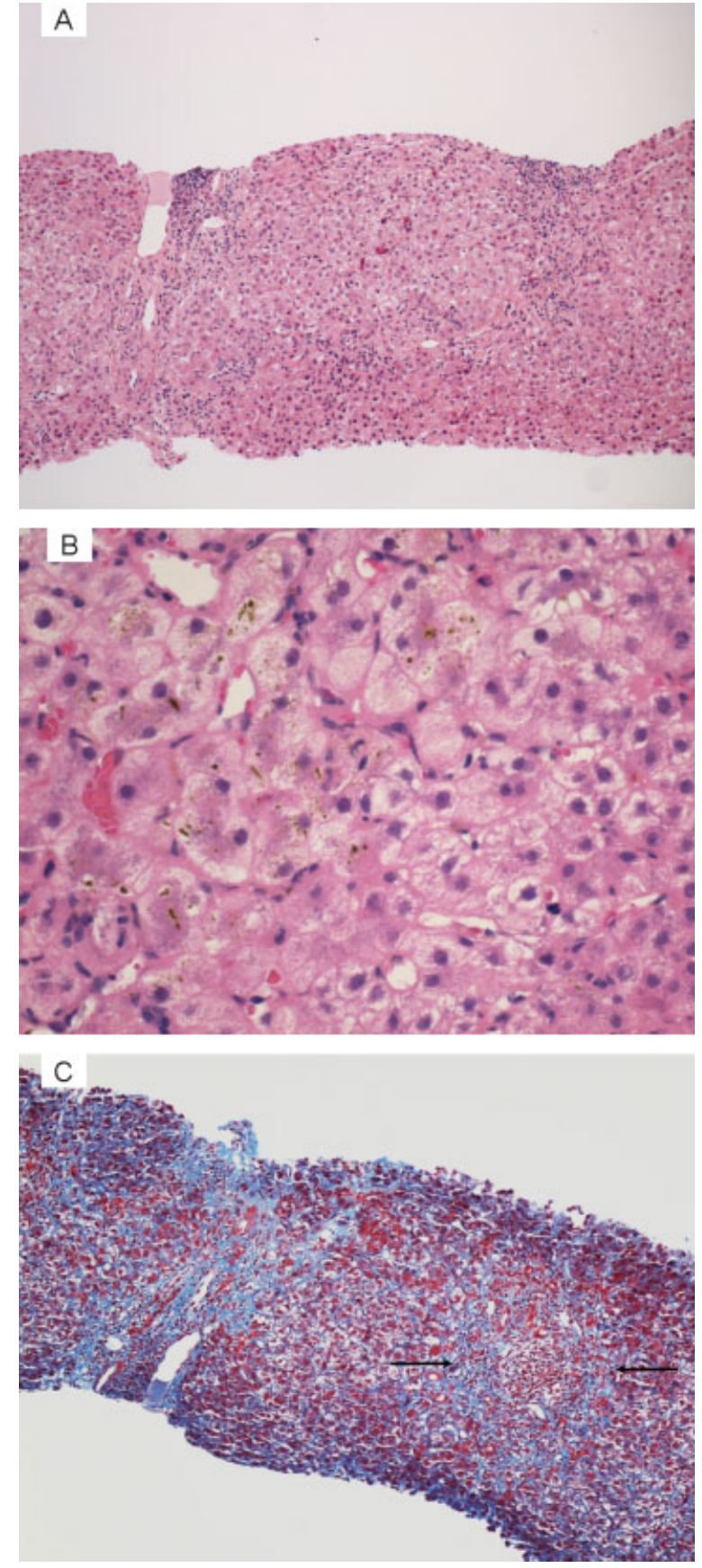

Fig. 2 Liver biopsy 12 months after liver transplantation. (A) Chronic hepatitis $C$ with moderate activity (hematoxylin and eosin [H\&E], $\mathrm{x} 100$ ). (B) Hepatocytic and canalicular cholestasis (H\&E, x400). (C) Extensive periportal fibrosis (arrows) and bridging fibrous septum on the left (Trichrome stain, $\mathrm{x} 100)$.

tolerability of treatment remains poor. ${ }^{13,14}$ Side effects, including severe anemia and rash, along with significant drug interactions with tacrolimus and cyclosporine limit their use in the post-LT setting. ${ }^{15,16}$ These issues may be especially apparent in patients with fibrosing cholestatic hepatitis who are often much sicker and more debilitated owing to the rapid progression of fibrosis and liver dysfunction. 

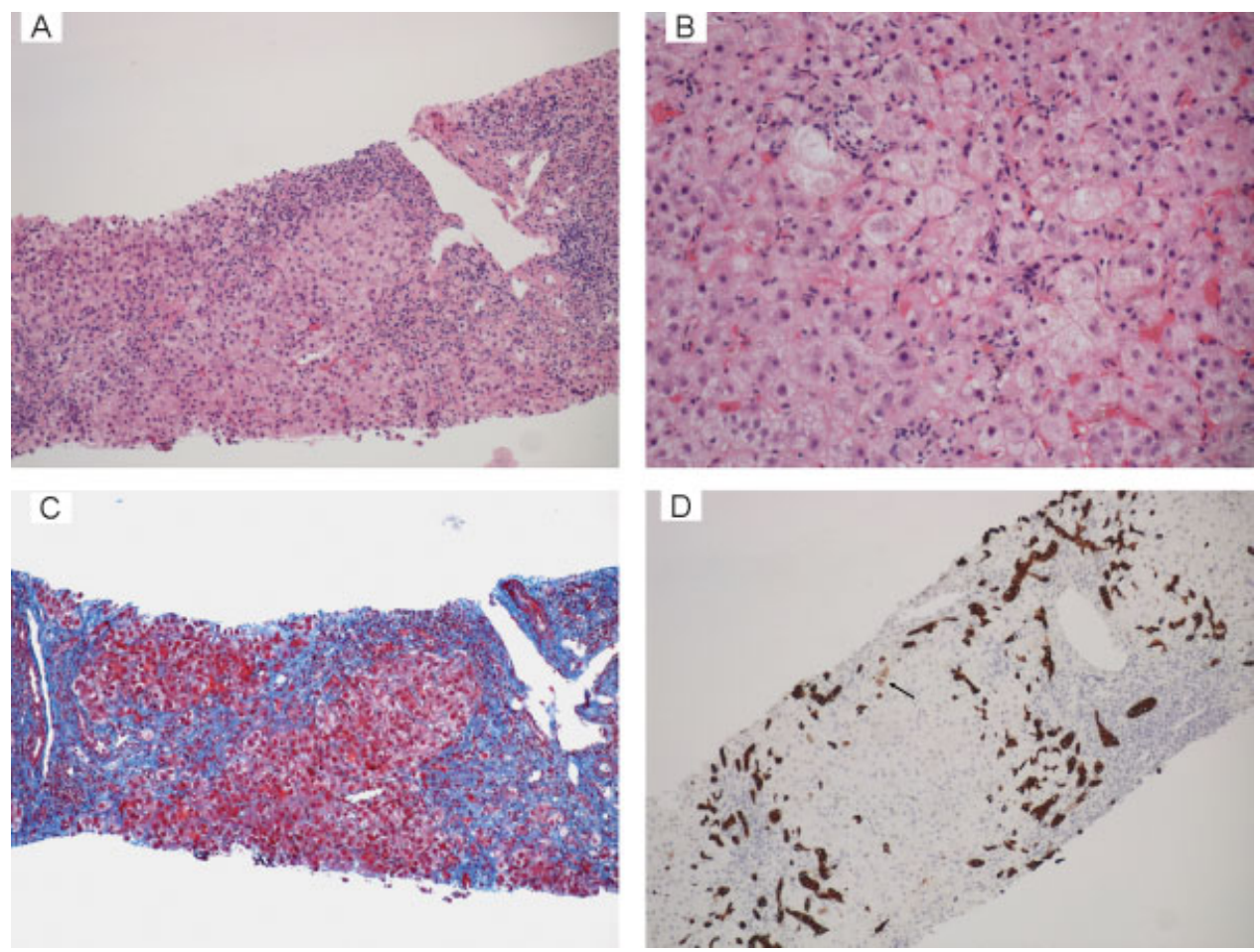

Fig. 3 Liver biopsy 15 months after liver transplantation. (A) Chronic hepatitis $C$ with moderate activity and bridging fibrous septa (hematoxylin and eosin [H\&E], x40). (B) Cholestasis with feathery degeneration of hepatocytes (H\&E, x200). (C) Extensive fibrous septum formation in transition to cirrhosis (Trichrome stain, x100). (D) Moderate ductular reaction and rare cholestatic hepatocytes (arrow) (CK7 immunostain).

After recurrence of his HCV, our patient had cholestasis and rapidly progressing fibrosis noted on his liver biopsies, consistent with $\mathrm{FCH}$. An attempt at treatment with pegylated interferon and ribavirin was poorly tolerated. Recent data has suggested that the combination of sofosbuvir and ribavirin may be effective in the treatment of HCV infection post-LT. ${ }^{17}$

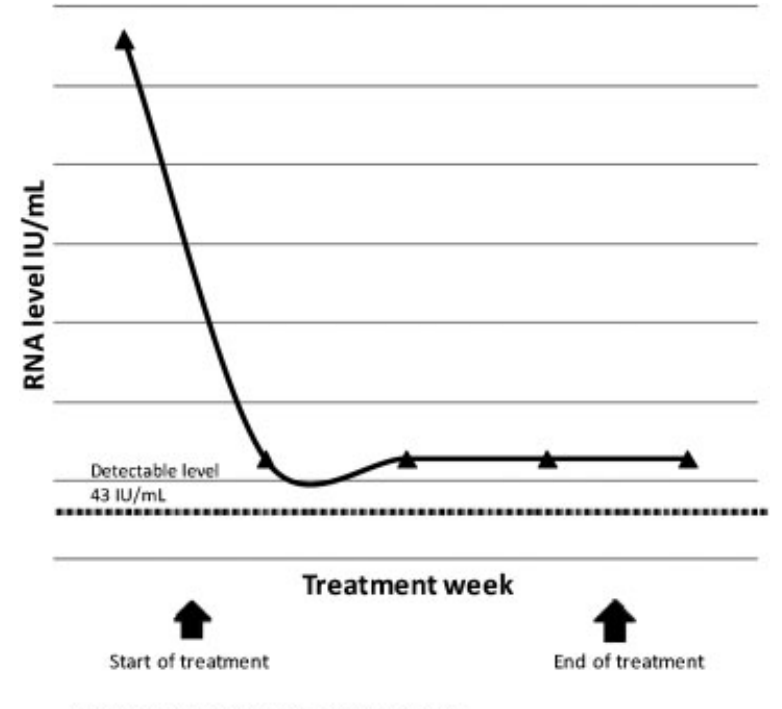

HCV, hepatitis C virus; RNA, ribonucleic acid

Fig. 4 Hepatitis C virus RNA level on sofosbuvir and ribavirin.
This case illustrates that the combination may also be effective in the most aggressive form of HCV recurrence with suppression of viremia and normalization of the patient's liver chemistry tests after 24 weeks. No interaction between the HCV treatment regimen and his immunosuppression was observed. The regimen was well tolerated without significant side effects.

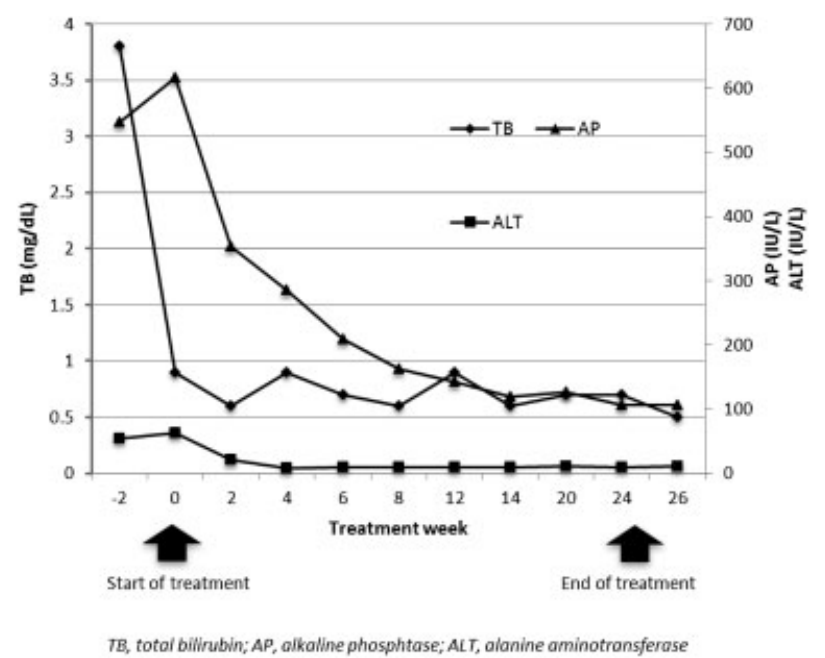

Fig. 5 Liver chemistry test on sofosbuvir and ribavirin. 


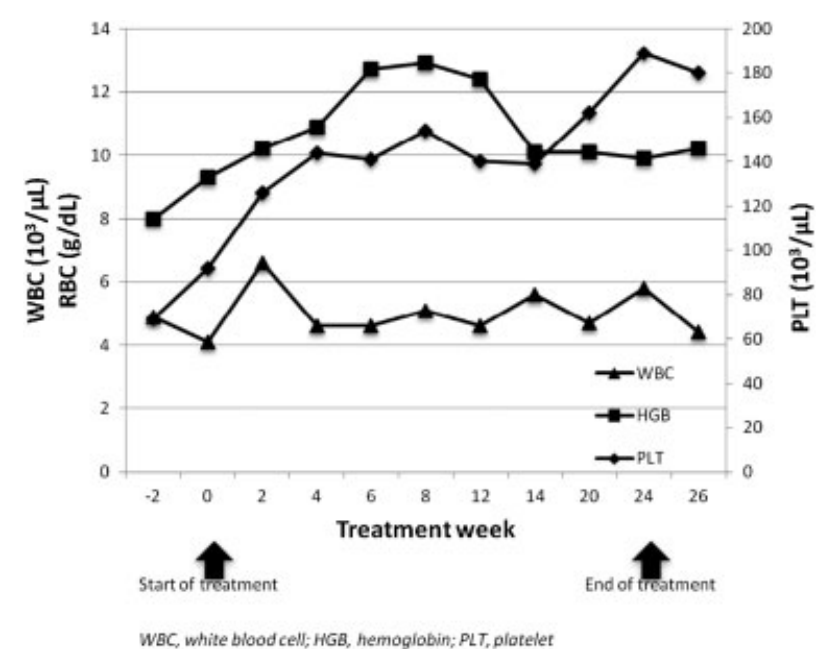

Fig. 6 Peripheral blood count on sofosbuvir and ribavirin.

\section{Conclusion}

Fibrosing cholestatic hepatitis or cholestatic variant of hepatitis $C$ is a rare, though uniformly serious, complication of HCV recurrence after liver transplant. The diagnosis of $\mathrm{FCH}$ is often followed by graft loss or death. Prior HCV regimens have had limited success in reversing this process. However, with the advent of the next-generation, direct-acting antivirals, like sofosbuvir, treatment of $\mathrm{FCH}$ may be feasible with increased efficacy and improved tolerability. A safe and efficacious treatment of FCH would have a significant impact on post-LT outcomes, as recurrent $\mathrm{HCV}$ remains the most common cause of graft loss and death. This case illustrates a successful treatment of FCH with a combination of sofosbuvir and ribavirin with dramatic normalization of liver enzyme activities and resolution of HCV related symptoms. Additional prospective studies with sofosbuvir-based HCV regimen will need to be studied in $\mathrm{FCH}$ to validate the promising findings of this case.

\section{References}

1 United Network for Organ Sharing. Transplant trends. Available at: http://www.unos.org/. Accessed November 1, 2013

2 Garcia-Retortillo M, Forns X, Feliu A, et al. Hepatitis C virus kinetics during and immediately after liver transplantation. Hepatology 2002;35(3):680-687

3 Gane EJ, Portmann BC, Naoumov NV, et al. Long-term outcome of hepatitis C infection after liver transplantation. N Engl J Med 1996; 334(13):815-820
4 Berenguer M, Prieto M, Rayón JM, et al. Natural history of clinically compensated hepatitis $C$ virus-related graft cirrhosis after liver transplantation. Hepatology 2000;32(4 Pt 1): 852-858

5 Cimsit B, Assis D, Caldwell C, et al. Successful treatment of fibrosing cholestatic hepatitis after liver transplantation. Transplant Proc 2011;43(3):905-908

6 Satapathy SK, Sclair S, Fiel MI, Del Rio Martin J, Schiano T. Clinical characterization of patients developing histologically-proven fibrosing cholestatic hepatitis $C$ post-liver transplantation. Hepatol Res 2011;41(4):328-339

7 Narang TK, Ahrens W, Russo MW. Post-liver transplant cholestatic hepatitis C: a systematic review of clinical and pathological findings and application of consensus criteria. Liver Transpl 2010;16(11):1228-1235

8 Gopal DV, Rosen HR. Duration of antiviral therapy for cholestatic HCV recurrence may need to be indefinite. Liver Transpl 2003; $9(4): 348-353$

9 Gane EJ, Stedman CA, Hyland RH, et al. Nucleotide polymerase inhibitor sofosbuvir plus ribavirin for hepatitis C. N Engl J Med 2013;368(1):34-44

10 Osinusi A, Meissner EG, Lee YJ, et al. Sofosbuvir and ribavirin for hepatitis $C$ genotype 1 in patients with unfavorable treatment characteristics: a randomized clinical trial. JAMA 2013;310(8): 804-811

11 Guillouche P, Féray C. Systematic review: anti-viral therapy of recurrent hepatitis $\mathrm{C}$ after liver transplantation. Aliment Pharmacol Ther 2011;33(2):163-174

12 Levitsky J, Fiel MI, Norvell JP, et al. Risk for immune-mediated graft dysfunction in liver transplant recipients with recurrent HCV infection treated with pegylated interferon. Gastroenterology 2012;142(5):1132-1139, e1

13 Werner CR, Egetemeyr DP, Lauer UM, et al. Telaprevir-based triple therapy in liver transplant patients with hepatitis $C$ virus: a 12 week pilot study providing safety and efficacy data. Liver Transpl 2012;18(12):1464-1470

14 Al Nahdi N, Ford JA, Greanya ED, et al. First successful treatment of post-liver transplant hepatitis $C$ fibrosing cholestatic hepatitis with boceprevir, peginterferon and ribavirin in a pre-transplant null responder. Ann Hepatol 2013;12(1):156-160

15 Garg V, van Heeswijk R, Lee JE, Alves K, Nadkarni P, Luo X. Effect of telaprevir on the pharmacokinetics of cyclosporine and tacrolimus. Hepatology 2011;54(1):20-27

16 Hulskotte E, Gupta S, Xuan F, et al. Pharmacokinetic interaction between the hepatitis $C$ virus protease inhibitor boceprevir and cyclosporine and tacrolimus in healthy volunteers. Hepatology 2012;56(5):1622-1630

17 Charlton MR, Gane EJ, Manns MP, et al. Sofosbuvir and Ribavirin for the treatment of established recurrent hepatitis $C$ infection after liver transplantation: preliminary results of a prospective, multicenter study. Paper presented at: 64th Annual Meeting of the American Association for the Study of Liver Diseases; November 1-5, 2013; Washington, DC 